\title{
The Cyclotron Resonance Scattering Features In Neutron Star Binaries Observed By INTEGRAL
}

\author{
Wei Wang \\ National Astronomical Observatories, Chinese Academy of Sciences, Beijing 100012, China \\ email: wangwei@bao.ac.cn
}

\begin{abstract}
Cyclotron resonance scattering features (CRSF) are the direct observational evidence for the strongly magnetized neutron stars. Since the first detection of the absorption line in the $\mathrm{X}$-ray source Her X-1 thirty years ago, more than ten sources are indentified as the strongly magnetized neutron stars through detecting CRSFs. INTEGRAL is the new X-ray/gamma-ray mission with good angular resolution, high sensitivity and spectral resolution in the range of 18 $C 200 \mathrm{keV}$, so that it provides us a good chance to detect the CRSFs in neutron star systems. INTEGRAL has confirmed the line features in 5 previous known sources and discovered 4 new candidates. Physical mechanism of CRSFs and accretion physics can be probed with detailed spectral analysis.
\end{abstract}

Keywords. stars: neutron, X-rays: binaries

\section{Introduction}

INTEGRAL is the ESA's operational space-based hard X-ray/soft gamma-ray telescope. There are two main instruments aboard INTEGRAL, the imager IBIS and the spectrometer SPI, supplemented by two X-ray monitors JEM-X and an optical monitor OMC. The co-aligned observations allow us to study X-ray pulsars in a wider energy band of $3-500 \mathrm{keV}$. The good spectral resolution and high sensitivity around tens of $\mathrm{keV}$ provide a good chance to search for the cyclotron resonance scattering features in neutron star accretion systems.

With the INTEGRAL all-sky survey observations, we have confirmed the previous reported CRSFs in several systems, like $4 \mathrm{U} 0115+63$ (the fundamental energy $E_{0} \sim 10-$ $15 \mathrm{keV}$, Li et al. 2012); Her X-1 ( $E_{0} \sim 39 \mathrm{keV}$, Klochkov et al. 2007); V 0332+53 ( $E_{0} \sim$ $26 \mathrm{keV}$, Kreykenbohm et al. 2005); A 0535+26 ( $E_{0} \sim 45 \mathrm{keV}$, Caballero et al. 2007); Vela X-1 $\left(E_{0} \sim 27 \mathrm{keV}\right.$, Schanne et al. 2007). In addition, INTEGRAL observations discovered four new candidates: $4 \mathrm{U} 2206+54\left(E_{0} \sim 30 \mathrm{keV}\right.$, Wang 2009); 2S 0114+65 $\left(E_{0} \sim 22 \mathrm{keV}\right.$, Bonning \& Falanga 2005); RX J0440.9+4431 $\left(E_{0} \sim 32 \mathrm{keV}\right.$, Tsygankov et al. 2012); and IGR J01583+6713 ( $E_{0} \sim 35 \mathrm{keV}$, Wang 2010a). Assuming the electron absorption line case, we can calculate the magnetic field strength of detected neutron star systems by using the the formula $\left[B / 10^{12} \mathrm{G}\right]=\left[E_{\text {cycl }} / 11.6 \mathrm{keV}\right](1+z)$, where $E_{\text {cycl }}$ is the energy of the fundamental line, here $E_{\mathrm{cycl}}=29.6 \mathrm{keV}$, and $z$ is the gravitational redshift near the surface of the neutron star. For a canonical neutron star of $1.4 M_{\odot}$ with a radius of $10 \mathrm{~km}$, we can take $z \sim 0.3$. Then we derived the magnetic field strength of the accreting magnetized neutron stars in the range of $(1-5) \times 10^{12} \mathrm{G}$. 


\section{Some Interesting Results}

Detailed spectral analysis of CRSFs and the variations can probe the accretion physics near the polar cap regions of X-ray pulsars in binaries. During the 2008 giant outburst, we determined the spin period of the neutron star in $4 \mathrm{U} 0115+63$ at $\sim 3.61430 \pm 0.00003 \mathrm{~s}$, and a spin up rate during the outburst of $\dot{P}=(-7.24 \pm 0.03) \times 10^{-6} \mathrm{~s} \mathrm{~d}^{-1}$ (Li et al. 2012). And the spectral analysis combined with JEM-X and IBIS confirmed the 5 cyclotron line harmonics in $4 \mathrm{U} 0115+63$ during the giant outburst. In addition, The fundamental absorption line energy varies during the outburst: around $15 \mathrm{keV}$ during the rising phase, and transiting to $\sim 10 \mathrm{keV}$ during the peak of the outburst, and further coming back to $\sim 15 \mathrm{keV}$ during the decreasing phase. The variations of photon index show the correlation with the fundamental line energy changes: the source becomes harder around the peak of the outburst and softer in both rising and decreasing phases. The possible correlation between X-ray luminosity and the fundamental line energy $E_{0}$ is also confirmed. The spectral transition around the peak phase of the outburst should be related to X-ray luminosity. When $L_{x}>6.76 \times 10^{37} \mathrm{ergs} \mathrm{s}^{-1}$, we detect the familiar fundamental CRSF at $E_{0} \approx 10 \mathrm{keV}$, below this luminosity level, the fundamental line energy changes to be $E_{0} \approx 15 \mathrm{keV}$.

We identified two cyclotron absorption features around $30 \mathrm{keV}$ and $60 \mathrm{keV}$ in a high mass X-ray binary 4U 2206+54 (Wang 2009). This special source contains a superslow pulsation neutron star with the spin period of $\sim 5560 \mathrm{~s}$ (Wang 2009, 2010b; Reig et al. 2009 ) and the neutron star shows a spin-down trend with a rate of $\sim 4.9 \times 10^{-7} \mathrm{~s} \mathrm{~s}^{-1}$ (Wang 2012; Finger et al. 2010). In the standard evolution model of X-ray binaries, this slow pulsation period $\left(P_{\text {spin }}>1000 \mathrm{~s}\right)$ cannot be reached except for the much stronger surface magnetic field. Combined with the very fast spin-down rate in the source, we suggested that $4 \mathrm{U} 2206+54$ could be an accreting magnetar which has a magnetic field higher than $10^{14}$ G! However, we also should re-consider the CRSFs in this source, if the features are explained as electron absorption lines, the derived magnetic field is $\sim 3 \times$ $10^{12} \mathrm{G}$; while in the proton absorption case, the derived magnetic field is about $10^{15} \mathrm{G}$. Thus the physical nature and origin of $4 \mathrm{U} 2206+54$ is still in dispute at present, requiring further studies.

\section{References}

Bonning, E. W. \& Falanga, M. 2005, A\&SA, 436, L31

Caballero, I. et al. 2007, A\& $A, 465$, L21

Klochkov, D. et al. 2007, arXiv:0704.3062

Kreykenbohm, I. et al. 2005, A\&SA, 433, L45

Li. J., Wang, W. \& Zhao, Y., 2012, MNRAS, 423, 2854

Reig, P. et al., 2009, A\& A, 494, 1073

Schanne, S. et al. 2007, Proc. 6th INTEGRAL Workshop The Obscured Universe, Moscow, 3-7 July 2006 (ESA SP-622)

Tsygankov, S. S., Krivonos, R. A., \& Lutovinov, A. A. 2012, MNRAS, 421, 2407

Wang, W. 2009, MNRAS, 398, 1428

Wang, W. 2010a, AESA, 516, A15

Wang, W. 2010b, A\&AA, 520, A22

Wang, W. 2012, in preparation 